\title{
The perception of emotion: Parallel neural processing of the affective and discriminative properties of opiates
}

\author{
GERARD M. MARTIN \\ Memorial University of Newfoundland, St. John's, Newfoundland, Canada \\ and \\ ANTOINE BECHARA and DEREK VAN DER KOOY \\ University of Toronto, Ontario, Canada
}

\begin{abstract}
In most theories of emotion and motivation, it is assumed that the processing of the perceptual (cognitive) and affective (noncognitive) properties of stimuli are interdependent. We modeled the relationship between the perceptual and affective components of emotional and motivational ex periences by using morphine-induced effects. Lesions and pharmacological manipulations that block the affective properties of opiates in place- and flavor-conditioning paradigms did not influence rats' treatment of morphine as a discriminative cue for learning. We suggest that the affective/noncognitive properties of stimuli that impart negative and positive valence to other events are processed separately from and in parallel to the perceptual/cognitive properties of these stimuli.
\end{abstract}

The terms emotion and motivation are generally thought of as describing the same phenomenon, even though the terminology used to discuss them differs (Bindra, 1969; Toates, 1986). Both emotion and motivation subsume two types of processes: perceptual/cognitive processes, which are subjective phenomena, and affective/noncognitive processes, which exist as a function of their ability to modify behavior. The discussion of humans is more often concerned with emotion, because of the distinct cognitive component that is introspectively available to us. In other animals, supposedly noncognitive/affective modifications of behavior are more often studied, and discussions usually center on motivation. In our experiments, we asked whether the unconditional effects of psychoactive drugs, which are powerful emotional and motivational stimuli, could be separated into a cognitive component (which can be perceptually identified and used as a signal by an organism) and a noncognitive component, which modifies the affective properties of other stimuli but is not used as a signal.

The distinction between cognitive and noncognitive components has been debated for years by those who have been interested in determining the necessary prerequisites for a full-fledged emotion or motivational experience. Some have argued that an emotional experience is the subsequent reflection of a purely noncognitive event, with the physiological changes that take place in response to

This research was supported by grants from the Natural Science and Engineering Research Council of Canada. We thank Mark Holder for helpful comments. Correspondence should be addressed to Gerard $\mathbf{M}$. Martin, Department of Psychology, Memorial University of Newfoundland, St. John's, Newfoundland A1B 3X9, Canada. external events being the actual emotion (e.g., James, 1884, 1890; Lange, 1967). Others have argued that both the perception of a situation and the concomitant physiological changes that take place occur simultaneously and produce emotion in response to subcortical changes (Cannon, 1927). These opposing interpretations of emotion have been combined in the view that a full-fledged emotion is the product of an interaction between the perception of a situation and a physiological change (Schacter \& Singer, 1962). It is thus believed that emotion is incomplete if either an appraisal of the situation or a physiological change is absent (Buck, 1986). However, this modern compromise position has its detractors. Zajonc $(1980,1984)$ has argued that emotion exists in the absence of any conscious perception, because emotions can be displayed by an individual who is totally unaware of the emotion or the precipitating event. Others have argued that an emotional experience is not possible in the absence of any conscious perception, although it may be possible to have conscious perception without emotion (Lazarus, 1982).

Historically, the treatment of specific motivational processes in animals has paralleled the treatment of emotion in humans. Initially it was suggested that particular peripheral physiological changes accounted for hunger and thirst (Cannon, 1918; Carlson, 1916). Subsequent work, however, revealed that specific peripheral physiological changes were not correlated with any particular motivational state. Moreover, bypassing sensory channels by intragastrically infusing food or fluid or stimulating sensory channels without producing physiological changes showed that neither event alone was sufficient to produce the full spectrum of demonstrable motivational changes 
usually seen in connection with food or fluid (Holman, 1968; Nicolaidis \& Rowland, 1974; Sterritt \& Smith, 1965).

The findings described above reveal that our understanding of emotion and motivation has been hampered by our inability to experimentally separate cognitive/perceptual components from affective/noncognitive components. In the case of psychoactive drug-induced states, such separation is possible if one assumes that there are valid animal models of the cognitive and noncognitive components of drug experiences. Like emotions and motivations, drugs that are self-administered consist of both affective and cognitive components. Drugs have the capacity to modify the affective value of events with which they are paired, and drugs also have cognitive or perceptual features that can be used to distinguish one druginduced state from another. Place- and flavor-conditioning procedures are used to measure the affective components of opiates. Rats demonstrate strong preferences for environments paired with opiate injections and paradoxically show aversions for flavors paired with the same injections (Bechara \& van der Kooy, 1985; Martin, Bechara, $\&$ van der Kooy, 1988). The aversions are due to opiate actions at peripheral receptors and can be blocked by pretreatment with methylnaltrexone, a peripheral opiate antagonist (Bechara, Zito, \& van der Kooy, 1987; Martin et al., 1988). The positive reinforcing effects are due to the opiate actions at specific receptor populations in the forebrain and midbrain (Mucha, van der Kooy, O'Shaughnessy, \& Bucenieks, 1982; Phillips \& LePiane, 1980; van der Kooy, Mucha, O'Shaughnessy, \& Bucenieks, 1982). Selective lesions of a brainstem output (the tegmental pedunculopontine nucleus) of these forebrain and midbrain systems abolishes the positive reinforcing effects of systemically administered opiates (Bechara \& van der Kooy, 1989). The cognitive or perceptual component of opiates is measured by their ability to gain control over a response, in our case the consumption of a novel-tasting vinegar solution (Martin, Gans, \& van der Kooy, 1990; Mastropaolo, Moskowitz, Dacaney, \& Riley, 1989). The perceptual or discriminative effects of the opiate state are used to predict whether or not the novel-tasting solution will be followed by injections of an aversive drug, lithium chloride $(\mathrm{LiCl})$.

In Experiment 1, we show that morphine has a cognitive/perceptual component, by demonstrating that rats can learn to withhold consumption in the presence of a morphine-induced state and to consume a saccharin solution when they are in a saline-induced state. Once the rats acquired the discrimination, the control exerted by morphine over fluid consumption was found to be mediated through opiate receptors because naloxone pretreatment caused the rats to treat a morphine trial like a saline trial. Using the peripheral opiate antagonist methylnaltrexone, we then demonstrated that morphine's control was not mediated by peripheral opiate receptors that are known to mediate the aversive properties of opiates. We also demonstrated that lesioning the tegmental pedunculopontine nuclei did not influence the capacity of morphine to control consumption, although such lesions prevent morphine from supporting place preferences. In Experiment 2, we extended the observations made in Experiment 1 by showing that acquisition of the discrimination was not affected by lesions of the tegmental pedunculopontine nuclei and pretreatment with methylnaltrexone. To the extent that one can use drug-induced states to model emotion/motivation, our data suggest that the affective and cognitive components considered essential for a fullfledged emotion or motivation exist independently of one another.

\section{EXPERIMENT 1}

\section{Method}

Subjects. Twenty-eight male Wistar rats obtained from Charles River were used.

Procedure. The deprivation regime used throughout the experiment was initiated 1 week after the animals had been familiarized with being housed singly in suspended wire cages in the laboratory. The deprivation regime consisted of $15 \mathrm{~min}$ of access to water between 0900 and $1000 \mathrm{~h}$ and $30 \mathrm{~min}$ of access to water between 1500 and $1600 \mathrm{~h}$ each day. All experimental manipulations were carried out during the 15-min drinking period.

Drug discrimination training was carried out over 10 days. Each 2-day period was a training cycle. On odd-numbered days, during discrimination training, all rats were injected subcutaneously (s.c.) with $5 \mathrm{mg} / \mathrm{kg}$ of morphine dissolved in $1 \mathrm{ml} / \mathrm{kg}$ of physiological saline $15 \mathrm{~min}$ prior to $15 \mathrm{~min}$ of access to a $2.0 \%$ vinegar solution ( $2 \mathrm{ml}$ of Heinz white vinegar in $98 \mathrm{ml}$ of tap water). Vinegar removal was followed by an immediate intraperitoneal (i.p.) injection of $130 \mathrm{mg} / \mathrm{kg}$ of $\mathrm{LiCl}$ dissolved in $3 \mathrm{ml}$ of distilled water. On even-numbered days, the discrimination group was injected s.c. with saline $15 \mathrm{~min}$ prior to $15 \mathrm{~min}$ of access to vinegar. Vinegar removal was followed by an immediate i.p. injection of $3 \mathrm{ml} / \mathrm{kg}$ of physiological saline.

Blocking all opiate receptors. We determined whether or not the discriminative effects of morphine were naloxone-reversible, in order to ensure that the rat's discrimination between morphine and saline trials was mediated through opiate receptors rather than through some nonspecific effect of our opiate injection. The 28 rats were randomly assigned to one of four groups. The four conditions were: an s.c. injection of saline followed immediately by an s.c. injection of morphine (SAL-MOR-VIN), an s.c. injection of $0.2 \mathrm{mg} / \mathrm{kg}$ of naloxone followed immediately by an s.c. injection of morphine (NAL-MOR-VIN), an s.c. injection of naloxone followed by an s.c. injection of saline (NAL-SAL-VIN), and an s.c. injection of saline followed by another s.c. injection of saline (SAL-SAL-VIN). All animals were allowed $15 \mathrm{~min}$ of access to vinegar 15 min after the second injection.

Blocking morphine's aversive effects. We determined whether the peripheral effects of opiates, which mediate the aversive properties of opiates, contributed to the discriminative control exerted by morphine over fluid consumption. We selected a dosage of methylnaltrexone that blocks the capacity of morphine to produce flavor aversions (Bechara et al., 1987). The rats used in the naloxone test were randomly reassigned to one of four new conditions on the day after the naloxone test. The four conditions were: an i.p. injection of saline followed immediately by an s.c. injection of morphine (SAL-MOR-VIN), an i.p. injection of $10 \mathrm{mg} / \mathrm{kg}$ of methylnaltrexone followed immediately by an s.c. injection of morphine 
(MN-MOR-VIN), an i.p. injection of methylnaltrexone followed immediately by an s.c. injection of saline (MN-SAL-VIN), and an i.p. injection of saline followed immediately by an s.c. injection of saline (SAL-SAL-VIN). All animals were given $15 \mathrm{~min}$ of access to vinegar $15 \mathrm{~min}$ after the second injection.

Retraining. All rats were given two additional training cycles after the methylnaltrexone test. The rats were then returned to ad-lib food and tap water.

Blocking morphine's positive reinforcing effects. We assessed the effect of selectively abolishing the positive reinforcing effects of opiates on the discriminative control exerted by morphine over vinegar consumption. The tegmental pedunculopontine nuclei were removed by injecting $2 \mu \mathrm{l}$ of a $2 \%$ ibotenic acid solution bilaterally (coordinates AP -7.8 posterior to bregma, $\mathrm{L} \pm 1.6 \mathrm{~mm}$ lateral to the midline, and DV -5.8 below the dura, with the mouthbar set at $-3.3 \mathrm{~mm}$ below the interaural line). Control animals were injected with the phosphate-buffered solution that was used to dissolve the ibotenic acid. Ten rats with histologically verified bilateral lesions of the tegmental pedunculopontine nuclei (Bechara \& van der Kooy, 1989) and 9 control rats were used in subsequent phases of the experiment.

The deprivation regime was initiated 1 week after the completion of surgery. Testing was initiated 5 days after the deprivation regime had been initiated. Five animals with tegmental pedunculopontine nucleus lesions and 5 control animals were injected s.c. with $5 \mathrm{mg} / \mathrm{kg}$ of morphine $15 \mathrm{~min}$ prior to presentation of the vinegar solution. The remaining animals in the lesioned $(n=5)$ and control $(n=4)$ groups were injected with physiological saline 15 min prior to vinegar presentation. On the next day, the animals that had been injected with morphine prior to the presentation of vinegar were injected with physiological saline $15 \mathrm{~min}$ prior to presentation of vinegar, while the animals that had been injected with morphine were injected with physiological saline 15 min prior to the presentation of vinegar.

The rats were trained and tested in a place-conditioning paradigm starting 2 days after the end of the morphine tests, in order to ensure that these ibotenic acid lesions of the tegmental pedunculopontine area did abolish the positive motivational properties of morphine, as we have previously reported (Bechara \& van der Kooy, 1989). All animals were given a 5 -min exposure to a gray box on the 1 st day. Over the next 6 days, place conditioning occurred. During this training, the animals were exposed to two square boxes that differed in color, texture, and smell: black walls, smoother Plexiglas floors, and the smell of $2 \%$ glacial acid, versus white walls, wood chip floors, and the smell of wood. On odd-numbered days, the rats were injected with $5 \mathrm{mg} / \mathrm{kg}$ of morphine s.c. prior to being placed into a white or a black box for $30 \mathrm{~min}$. On even-numbered days, the animals were injected s.c. with physiological saline prior to being placed into white or black boxes for $30 \mathrm{~min}$. Five animals in the lesioned and control groups were exposed to the black box on odd-numbered days and white boxes on even-numbered days. The remaining 5 lesioned and 4 control animals were exposed to white boxes on odd-numbered days and black boxes on evennumbered days. Testing was carried out 1 day after the last training day. The uninjected animals were placed in a large rectangular box with the two training environments on opposite sides, separated by a gray area with a grid floor. The rats were placed in the gray area and the amounts of time (in seconds) spent in the two treatment environments over the next $10 \mathrm{~min}$ were measured.

\section{Results}

Acquisition of the discrimination (i.e., drinking the novel-tasting solution only after saline injections) is presented in panel A of Figure 1; these results replicate earlier findings obtained when a variety of control groups for nonassociative effects were included (Martin et al., 1990). A $5 \times 2$ analysis of variance (ANOVA) revealed a significant cycles $\times$ days interaction $[F(4,108)=24.82$, $p<.01]$. Newman-Keuls tests revealed that the animals had acquired the discrimination by Cycles 4 and 5 , in that they consumed reliably more vinegar on the saline days than on the 2 morphine days ( $p s<.01$ ).

The discriminative effects of morphine were naloxonereversible, since the animals that were injected with naloxone prior to the injection of morphine treated the trial like a saline trial (Figure 1, panel B). A one-way ANOVA over the four groups presented in panel B of Figure 1 revealed a significant difference $[F(3,24)=54.01$, $p<.01]$. Newman-Keuls tests revealed that three groups drank reliably more vinegar than the one group injected with saline and morphine prior to the presentation of vine$\operatorname{gar}(p s<.01)$. Hence naloxone, at a dosage that would not have a large effect on fluid consumption in normal rats (Yirmiya \& Holder, 1987), but that would reverse all the opiate-mediated effects of morphine, eliminated the discriminative effects of morphine.

The discriminative effects of morphine were not methylnaltrexone-reversible, thereby indicating that the peripheral opiate receptors, which mediate the aversive effects of opiates, were not mediating the discrimination (Figure 1, panel C). A one-way ANOVA across the four groups presented in panel $\mathrm{C}$ revealed a significant difference $[F(3,24)=14.41, p<.01]$. Newman-Keuls tests revealed that the two groups of animals injected with morphine (whether methylnaltrexone-treated or not) drank reliably less than did the animals that were not injected with morphine.

Our findings show that morphine working through specific opiate receptors, rather than some nonspecific drug effect, mediates the discriminative control seen. The elimination of morphine's discriminative control when naloxone was injected prior to the injection of morphine showed that the discriminative effects of morphine are mediated through opiate receptors. We conclude that the opiate receptors mediating the discriminative effects are in the central nervous system, because the peripheral opiate receptors responsible for producing the aversive properties of opiates do not mediate the discriminative effects of morphine. Methylnaltrexone blocked the receptors responsible for the aversive effects and did not affect the capacity of morphine to control consumption in the discrimination paradigm.

Vinegar consumption by the 19 rats during a retraining cycle revealed that the discrimination was still intact (Figure 1, panel D). A $2 \times 2$ ANOVA (cycles $\times$ days) revealed a reliable days effect $[F(1,17)=601.12$, $p<.01]$, which reflects the discrimination.

Elimination of the reinforcing effects of morphine with lesions of the tegmental pedunculopontine nucleus did not interfere with the capacity of morphine to control vinegar consumption (Figure 1, panel E). A two-way ANOVA (lesioned or not lesioned $\times$ morphine or saline) 


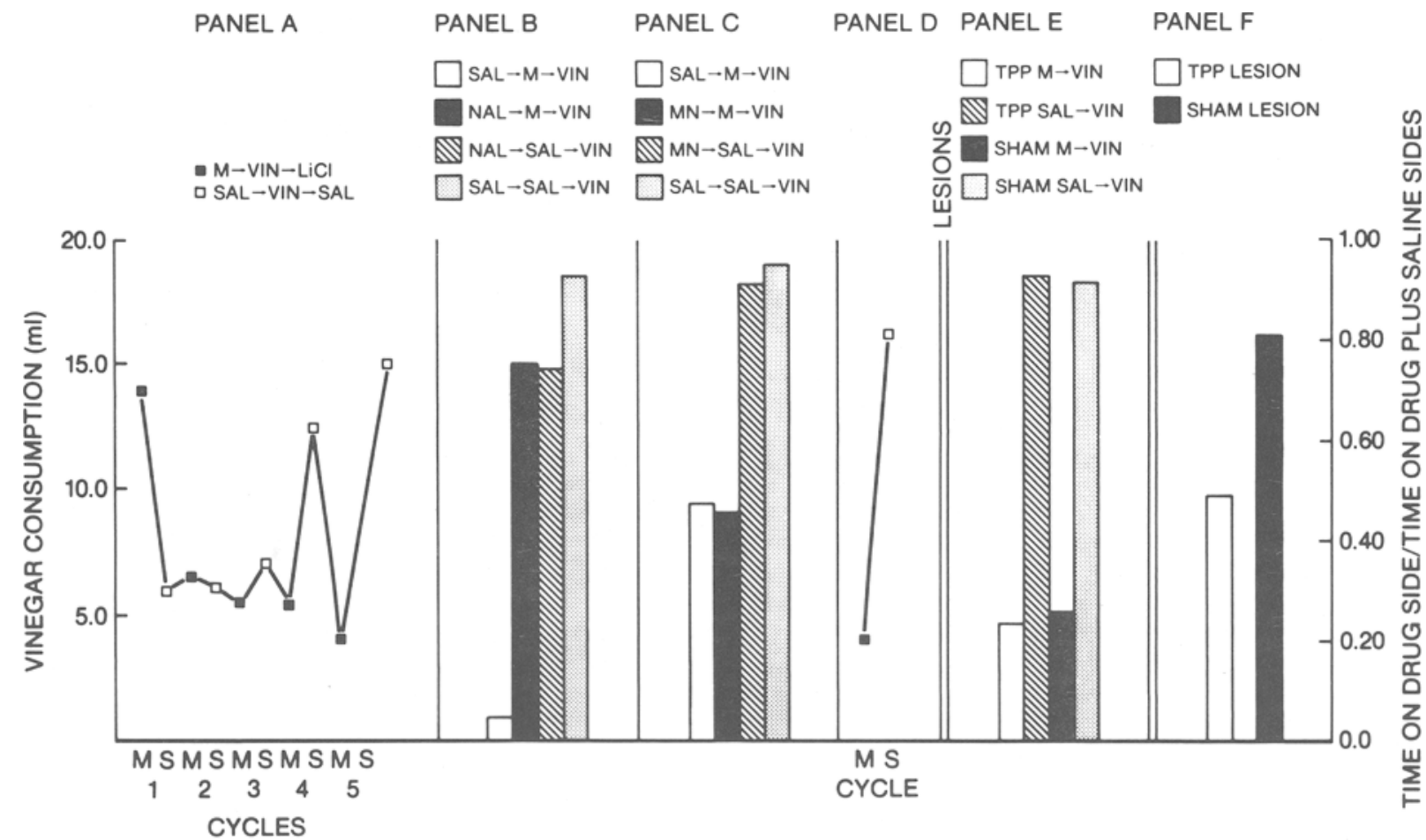

Figure 1. All fluid measures presented in this figure had standard errors of $1.94 \mathrm{ml}$ or less. (A) Vinegar consumption by rats during the initial five training cycles when animals were injected with morphine (M) 15 min prior to presentations of a vinegar solution (VIN) whose removal was followed by an injection of lithium chloride (LiCl). On alternate days, saline (S or SAL) was substituted for morphine and LiCl. By Cycles 4 and 5, animals had acquired the discrimination. (B) Vinegar consumption by the rats after being injected with saline (SAL), morphine (M), and/or naloxone (NAL). (C) Vinegar consumption by the rats after being injected with saline (SAL), morphine (M), and/or methylnaltrexone (MN). (D) Vinegar consumption by 19 of the rats during a retraining cycle in which morphine (M) was injected prior to presentation of vinegar, whose removal was followed by an injection of LiCl. Saline was substituted for morphine and LiCl on the S day. (E) Vinegar consumption by the same 19 rats after either control lesions (SHAM) or bilateral lesions of the tegmental pedunculopontine nucleus (TPP) when morphine (M) or saline (SAL) was injected prior to the presentation of the vinegar (VIN). (F) Place preference ratios for animals that had control lesions (SHAM) or bilateral lesions of the tegmental pedunculopontine nucleus (TPP).

over the vinegar consumption scores revealed a significant difference between morphine and saline trials $[F(1,15)=76.92, p<.01]$ and no other differences.

The ibotenic acid lesions of the tegmental pedunculopontine nucleus did eliminate the reinforcing effects of opiates (Figure 1, panel F). The rats that had ibotenic acid lesions did not show a place preference, whereas the rats with sham lesions did show a place preference $[t(17)$ $=2.39, p<.01]$.

\section{EXPERIMENT 2}

Experiment 1 revealed that neither the aversive nor the positive motivational properties of opiates are necessary for the maintenance of the discrimination. It remains possible that either the aversive or the positive motivational properties, in the absence of the other, are sufficient to mediate the discriminative effects of opiates. Furthermore, it is conceivable that the motivational effects of opiates are necessary to acquire, but not to maintain, the discrimination. Therefore, Experiment 2 was undertaken in an at- tempt to train a morphine discrimination in the absence of both the aversive (methylnaltrexone pretreatment) and positive motivational (tegmental pedunculopontine lesions) properties of opiates.

\section{Method}

Subjects. Nine naive male Wistar rats that were obtained from Charles River were used.

Procedure. All rats received bilateral ibotenic acid lesions of the tegmental nuclei 1 week after arrival in the laboratory, according to the procedure described in Experiment 1. Adaptation to the waterdeprivation regime used in the previous experiment commenced 2 weeks after the lesions were completed. Six training cycles started on the 8th day of water deprivation. On the odd-numbered training days of each cycle, the animals were injected i.p. with $5 \mathrm{mg} / \mathrm{kg}$ of methylnaltrexone, 1 min prior to an s.c. injection of $5 \mathrm{mg} / \mathrm{kg}$ of morphine. The animals were allowed $15 \mathrm{~min}$ of access to a $2.0 \%$ vinegar solution, 15 min after the morphine injection. Removal of the vinegar was followed by an i.p. injection of $130 \mathrm{mg} / \mathrm{kg}$ of $\mathrm{LiCl}$. On the even-numbered days of each cycle, the animals were again permitted $15 \mathrm{~min}$ of access to vinegar and a $0.4-\mathrm{ml}$ injection of physiological saline was substituted for each injection that was given on odd-numbered days. 


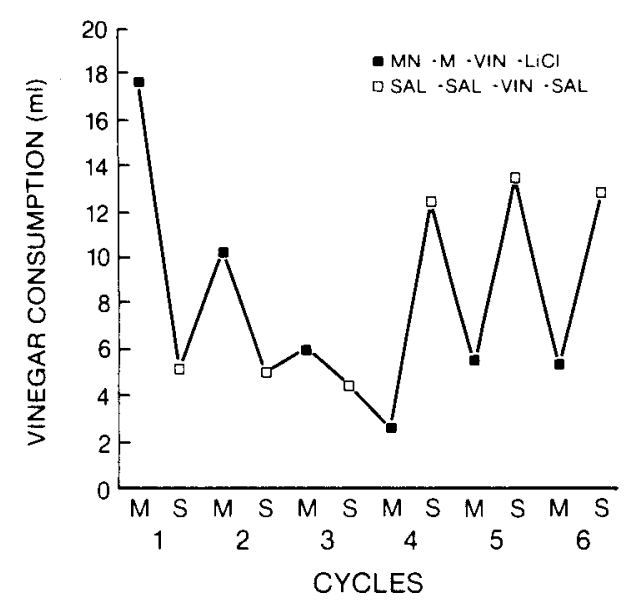

Figure 2. All fluid measures presented in this figure had standard errors of $1.66 \mathrm{ml}$ or less. Acquisition of morphine-discriminated vinegar consumption by rats that had bilateral lesions of the tegmental pedunculopontine nucleus and were injected with methylnaltrexone (MN) and morphine (M) prior to vinegar consumption on $M$ days. Saline (S or SAL) was substituted for MN and $M$ on $S$ days. Lithium chloride $(\mathrm{LiCl})$ was injected after vinegar removal on $\mathrm{M}$ days and saline was injected after vinegar removal on $S$ days.

\section{Results}

Neither the aversive motivational nor the positive motivational properties of opiates are necessary for the acquisition of a morphine discrimination (Figure 2). A two-way ANOVA revealed a significant cycles $\times$ days interaction $[F(5,40)=20.79, p<.01]$. Newman-Keuls tests revealed that animals were drinking reliably more vinegar on safe days than on training days $(p s<.01)$ by the fourth cycle. Indeed, the initial consumption of the methylnaltrexone-treated rats that had TPP lesions was comparable to that of unmanipulated rats, and they acquired the morphine discrimination as rapidly as the unmanipulated rats did (Figure 1, panel A). Again, only lesioned rats with histologically verified bilateral lesions of the tegmental pedunculopontine nuclei were included in the analysis. In addition, the behavioral effectiveness of the lesions was assessed after the discrimination training in 8 of the 9 rats employed, by testing the ability of $1.0 \mathrm{mg} / \mathrm{kg}$ of amphetamine s.c. to condition place preferences in a procedure identical to the conditioning of morphine place preferences as described in Experiment 1. As we have previously reported (Bechara \& van der Kooy, 1989), the tegmental-pedunculopontine-nuclei-lesioned rats did not show amphetamine-produced place preferences relative to the preferences shown by control rats $[t(14)=2.21, p<.01]$.

\section{GENERAL DISCUSSION}

The present experiments demonstrate that the affective properties of opiate action can be removed while the discriminative capacity of the drug-induced state to control responding is left intact. In particular, blockade of the peripheral opiate receptors, which mediate the aversive effects of opiates, and lesions of the tegmental pedunculopontine nuclei, which are essential for demonstrating the positive effects of opiates, did not influence retention (Experiment 1) or acquisition (Experiment 2) of a discrimination based on morphine. These data indicate that the cognitive properties of a drug state are not isomorphic with its affective properties. Moreover, the nervous system appears to process the cognitive and affective properties of opiates through parallel and noninteracting pathways. Conscious perceptual experiences may not come affectively prelabeled, although they may become associated with motivational effects that reliably occur at the same time. Zajonc (1980) has proposed a somewhat similar independence of affect and cognition, primarily on the basis of findings in studies of humans. For example, affective decisions, such as liking particular shapes because of preexposure to them, can be changed even though the subjects have no conscious memory of having been preexposed to the shapes. Others have proposed a similar independence of affect and cognition, on the basis of animal studies in which the formation of flavor aversions has not appeared to be dependent on cognitive appraisal of the situation (Garcia \& Holder, 1985).

The present demonstration that the affective properties of opiates are not essential to morphine's serving as a signal complements other attempts to understand the role of the affective component of motivation in behavior. Our data suggest that the affective properties of motivational stimuli are not necessary for the use of a drug as a cue by animals, and the work of other investigators indicates that the affective properties of motivational stimuli are not sufficient for the use of a drug as a cue by animals. Motivational cues dramatically influence behavior, as is evidenced by their effects on activity and by their effects on conditioned preferences or aversions for places and tastes. However, the role of motivational cues does not appear to include a signal function. Relief from hunger and thirst, in the absence of salient sensory cues (such as oropharyngeal stimulation), has produced poor control over behavior (Holman, 1968; Sterritt \& Smith, 1965). Animals have frequently failed to change instrumental responses when their motivational state has been changed (Dickinson \& Dawson, 1987), and animals have not withheld contact with aversive foods even though their motivational state could be used as a signal that the food was aversive (Weingarten \& Martin, 1989).

Our findings suggest that we should think about the affective properties of drugs as inaccessible features that can impart positive or negative valence to an event, but which cannot be used as perceptually distinct signals. We are proposing that the cognitive/perceptual properties of emotion are processed by the nervous system in a noninteracting manner, in parallel to the processing of the noncognitive/affective properties of motivational stimuli. Certainly, these separate cognitive and affective properties occur at the same time and may come to predict one another if elicited by the same external stimulus, but this 
full-fledged emotional experience would be a by-product of their learned association over time and not of their initial processing by the brain.

\section{REFERENCES}

BechARA, A., \& VAN DER KOOY, D. (1985). Opposite motivational effects of endogenous opioids in brain and periphery. Nature, 314, 533-534.

Bechara, A., \& van der Kooy, D. (1989). The tegmental pedunculopontine nucleus: A brainstem output of the limbic system critical for the conditioned piace preferences produced by morphine and amphetamine. Journal of Neuroscience, 9, 3400-3409.

Bechara, A., Zito, K. A., \& VAN DER Kooy, D. (1987). Peripheral receptors mediate the aversive conditioning effects of morphine in the rat. Pharmacology, Biochemistry \& Behavior, 28, 219-225.

BindRA, D. (1969). A unified interpretation of emotion and motivation. Annals of the New York Academy of Sciences, 159, 1071-1083.

Buck, R. (1986). The psychology of emotion. In J. E. Ledoux \& W. Hirst (Eds.), Mind and brain (pp. 275-300). New York: Cambridge University Press.

Cannon, W. B. (1918). The physiological basis of thirst. Proceedings of the Royal Society of London, 908, 283-301.

Cannon, W. B. (1927). The James-Lange theory of emotions: A critical examination and an alternative theory. American Journal of Psychology, 39, 106-224.

Carlson, A. J. (1916). The control of hunger in health and disease. Chicago: University of Chicago Press.

Dickinson, A., \& DAwson, G. R. (1987). The role of the instrumental contingency in the motivational control of performance. Quarterly Journal of Experimental Psychology, 39B, 77-93.

Garcia, J., \& Holder, M. D. (1985). Time, space and value. Human Neurobiology, 4, 81-89.

Holman, G. L. (1968). Intragastric reinforcement effect. Journal of Comparative \& Physiological Psychology, 69, 432-441.

JAMES, W. (1884). What is emotion? Mind, 9, 188-204.

JAMES, W. (1890). Principles of psychology. New York: Holt.

LANGE, C. (1967). Om Leudsbeveegelser (I. A. Haupt, Trans.). In K. Dunlap (Ed.), The emotions (pp. 33-77). Baltimore: Williams \& Wilkins. (Original work published 1922)

LAZARUS, R. B. (1982). Thoughts on the relations between emotion and cognition. American Psychologist, 37, 1019-1024.

Martin, G. M., Bechara, A., \&an der Kooy, D. (1988). Morphine preexposure attenuates the aversive properties of opiates without preexposure to the aversive properties. Pharmacology, Biochemistry \& Behavior, 30, 687-692.

Martin, G. M., Gans, M., \& Van der Kooy, D. (1990). Discriminative properties of morphine that modulate associations between tastes and lithium chloride. Journal of Experimental Psychology: Animal Behavior Processes, 16, 56-68.

Mastropaolo, J. P., Moskowitz, K. H., Dacaney, R. J., \& Riley, A. L. (1989). Conditioned taste aversions as a behavioral baseline for drug discrimination learning: An assessment with phencyclidine. Pharmacology, Biochemistry \& Behavior, 32, 1-8.

Mucha, R. F., van der Kooy, D., O'Shaughnessy, M., \& BuceNIEKS, P. (1982). Drug reinforcement studies by the use of place conditioning in rats. Brain Research, 243, 91-105.

Nicolaidis, S., \& Rowland, N. (1974). Self intravenous "drinking" in the rat. Journal of Comparative \& Physiological Psychology, 87, 1-15.

Phillips, A. G., \& LePiane, F. G. (1980). Reinforcing effects of morphine microinjections into the ventral tegmental area. Pharmacology, Biochemistry \& Behavior, 12, 965-968.

SChaCter, S., \& Singer, J. (1962). Cognitive, social, and physiological determinants of emotional state. Psychological Review, 69, 379-399.

Sterritt, G. M., \& SMIth, M. P. (1965). Reinforcement effects of specific components of feeding in young leghorn chicks. Journal of Comparative \& Physiological Psychology, 59, 171-175.

Toates, F. (1986). Motivational systems. Cambridge, U.K.: Cambridge University Press.

van der Kooy, D., Mucha, R. F., O'Shaughnessy, M., \& BuceNIEKS, P. (1982). Reinforcing effects of brain microinjections of morphine revealed by conditioned place preferences. Brain Research, 243, $107-117$.

Weingarten, H. P., \& Martin, G. M. (1989). Mechanisms of conditioned meal initiation. Physiology \& Behavior, 45, 735-740.

YIRMIYA, R., \& HoldeR, M. D. (1987). Effects of naloxone and cholecystokinin on food and water intake in vasopressi-deficient rats (Brattleboro strain). Peptides, 8, 763-767.

ZaJONC, R. B. (1980). Feeling and thinking: Preferences need no inferences. American Psychologist, 35, 151-175.

Zajonc, R. B. (1984). On the primacy of affect. American Psychologist, 39, 117-123.

(Manuscript received March 5, 1990; revision accepted for publication November 13, 1990.) 